\title{
RECURSIVE FUNCTIONS AND REGRESSIVE ISOLS
}

\section{J. BARBACK}

\section{Introduction.}

The reader is assumed to be familiar with the concepts and main results of the papers listed as references. We shall use the following notations:

$$
\begin{aligned}
\varepsilon & =\text { the set of all non-negative integers (numbers) } \\
\Lambda & =\text { the collection of all isols, } \\
\Lambda^{*} & =\text { the collection of all isolic integers. }
\end{aligned}
$$

Myhill [5] associated with every recursive combinatorial function $f(x)$ a function $F(X)$ from $\Lambda$ into $\Lambda$, called the canonical extension of $f(x)$; we shall write $C_{f}(X)$ for the canonical extension of $f(x)$. Nerode [8] associated with every recursive function $f(x)$ a function $F(X)$ from $\Lambda$ into $\Lambda^{*}$; we shall write $D_{f}(x)$ for Nerode's extension of $f(x)$. In the special case that $f(x)$ is recursive and combinatorial, $D_{f}(x)=C_{f}(X)$ for $X \in \Lambda$. It was proved in [7] that for a recursive function $f(x)$, the function $D_{f}(X)$ maps $\Lambda$ into $\Lambda$ if and only if $f(x)$ is eventually combinatorial. Regressive isols were introduced in [1]. Let $\Lambda_{R}$ denote the collection of all regressive isols. It is known that $\varepsilon \subset \Lambda_{R} \subset \Lambda$, where both $\Lambda_{R}-\varepsilon$ and $\Lambda-\Lambda_{R}$ have the cardinality of the continuum.

This paper deals with the following problem:

Which recursive functions $f(x)$ have the property that $D_{f}(X)$ maps $\Lambda_{R}$ into $\Lambda_{R}$ ?

A function $f(x)$ from $\varepsilon$ into $\varepsilon$ will be called increasing if

$$
x<y \Rightarrow f(x) \leqq f(y), \quad \text { for } x, y \in \varepsilon ;
$$

and eventually increasing if for some number $n$, the function $g(x)=$ $f(x+n)$ is increasing. The main result of this paper is as follows. For a recursive function $f(x)$ the function $D_{f}(X)$ maps $\Lambda_{R}$ into $\Lambda_{R}$ if and only if $f(x)$ is eventually increasing.

Received April 4, 1964.

This paper was written while the author was a student of Professor J. C. E. Dekker at Rutgers University. Research was supported by the U.S. National Science Foundation. 
Our presentation will make use of the theory of infinite series of isols. Infinite series of isols were introduced and studied in [1].

\section{Regressive isols.}

We shall here recall the concepts of a regressive function, regressive set and regressive isol.

Definitions. A function $t_{n}$ from $\varepsilon$ into $\varepsilon$ is regressive if it is one-to-one and there exists a partial recursive function $p(x)$ such that

$$
\begin{gathered}
\varrho t \subset \delta p, \\
p\left(t_{0}\right)=t_{0} \quad \text { and } \quad(\forall n)\left[p\left(t_{n+1}\right)=t_{n}\right] .
\end{gathered}
$$

A set is regressive, if it is finite or the range of a regressive function. An isol $T$ is regressive, if it contains at least one regressive set.

For every partial recursive function $f(x)$ with $\varrho f \subset \delta f$, let

$$
f^{0}(x)=x, \text { and } f^{n+1}(x)=f\left(f^{n}(x)\right) .
$$

Let $t_{n}$ be a regressive function. By $[1, \mathrm{pp} .80,81]$ there exists a partial recursive function $p(x)$ which satisfies, besides (1) and (2) the conditions

$$
\begin{gathered}
\varrho p \subset \delta p, \\
(\forall x)\left[x \in \delta p \Rightarrow(\exists n)\left[p^{n+1}(x)=p^{n}(x)\right]\right] .
\end{gathered}
$$

Definition. Every partial recursive function satisfying (1), (2), (3) and (4) is called a regressing function of $t_{n}$.

Notation. Let $p(x)$ be a partial recursive function satisfying (3) and (4). Then

$$
p^{*}(x)=(\mu y)\left[p^{y+1}(x)=p^{y}(x)\right], \quad \text { for } x \in \delta p .
$$

We note that in the event $p(x)$ is a regressing function of $t_{n}$, then $p^{*}(x)$ is a partial recursive function with

$$
\delta p^{*}=\delta p, \varrho t \subset \delta p^{*} \quad \text { and } \quad(\forall n)\left[p^{*}\left(t_{n}\right)=n\right] .
$$

\section{3. $\Sigma_{T} a_{n}$.}

We shall here recall the definition given in [1] of the infinite series $\Sigma_{T} a_{n}$, in case $a_{n}$ is a recursive function of $n$ and $T$ a regressive isol.

Notations. Let $j, k$ and $l$ denote the well-known recursive functions defined by 


$$
\begin{aligned}
j(x, y) & =x+\frac{1}{2}(x+y)(x+y+1), \\
j(k(n), l(n)) & =n .
\end{aligned}
$$

For any number $n, v(n)=\{x \mid x<n\}$.

For any number $n$ and sets $\delta$ and $\sigma$,

$$
j(n, \sigma)=\{j(n, y) \mid y \in \sigma\} \text { and } j(\delta, \sigma)=\{j(x, y) \mid x \in \delta \& y \in \sigma\} .
$$

We note that the function $j$ maps $\varepsilon^{2}$ one-to-one onto $\varepsilon$.

Definition. Let $a_{n}$ be any recursive function of $n$ and $T$ any regressive isol. If $T$ is finite, say $T=k$,

If $T$ is infinite,

$$
\sum_{T} a_{n}=\sum_{n<k} a_{n} \quad(0 \text { for } k=0) .
$$

$$
\sum_{T} a_{n}=\operatorname{Req} \sum_{0}^{\infty} j\left(t_{n}, v\left(a_{n}\right)\right),
$$

where $t_{n}$ is any regressive function ranging over any set in $T$.

By [1, Proposition 3] $\Sigma_{T} a_{n}$ depends on the regressive isol $T$ and not on the particular regressive function whose range is in $T$. Also, $\Sigma_{T} a_{n}$ is always an isol [1, Theorem 1] and in fact we shall prove in $\S 4$ that it is always a regressive isol.

Definition. Let $a_{n}$ be any function from $\varepsilon$ into $\varepsilon$. The function $s(n)$ such that

$$
\begin{aligned}
& s(0)=0, \\
& s(n)=\sum_{i<n} a_{i}, \quad \text { for } n>0,
\end{aligned}
$$

is the partial sum function of $a_{n}$.

One can easily verify that if the function $a_{n}$ is recursive and combinatorial then so is its partial sum function $s(n)$. The main result of [1] is Theorem A, stated below. It is essentially this theorem which enables us in $\S 5$ to express $D_{f}(T)$ (where $f$ is a recursive function and $T$ a regressive isol) as the difference of two infinite series each summed with respect to the regressive isol $T+1$.

Theorem A. [1, p. 86] Let $a_{n}$ be a recursive combinatorial function and $s(n)$ its partial sum function. Then, for every regressive isol $T$,

$$
\sum_{T} a_{n}=C_{s}(T) \text {. }
$$

\section{Fundamental properties of $\Sigma_{T} a_{n}$.}

Notation. Parentheses will be omitted according to the rule that association is to the right. Thus $f g(x)$ means the same as $f(g(x))$. 
THEOREM 1. For every recursive function $a_{n}, \Sigma_{T} a_{n}$ is a function from $\Lambda_{R}$ into $\Lambda_{R}$.

Proof. Let $T$ be any regressive isol. We have already noted that $\Sigma_{T} a_{n}$ is an isol and therefore we restrict our attention here to proving $\Sigma_{T} a_{n}$ is regressive. If either $T$ is finite or $a_{n}$ is identically zero from some number on, then $\Sigma_{T} a_{n}$ is finite and therefore regressive. Now assume $T$ is infinite and $a_{n}$ is positive infinitely often. Let $t_{n}$ be any regressive function ranging over any set in $T$. Let $f(x)$ denote the strictly increasing recursive function ranging over the set $\left\{n \mid a_{n}>0\right\}$. Then

$$
\begin{aligned}
& \sum_{0}^{\infty} j\left(t_{n}, v\left(a_{n}\right)\right) \in \sum_{T} a_{n}, \\
& \sum_{0}^{\infty} j\left(t_{n}, v\left(a_{n}\right)\right)=\sum_{0}^{\infty} j\left(t_{f(n)}, v\left(a_{f(n)}\right)\right) .
\end{aligned}
$$

We wish to prove $\Sigma_{T} a_{n}$ is regressive. We proceed to show that

$$
j\left(t_{f(0)}, 0\right), \ldots, j\left(t_{f(0)}, a_{f(0)}-1\right), j\left(t_{f(1)}, 0\right), \ldots, j\left(t_{f(1)}, a_{f(1)}-1\right), \ldots
$$

represents a regressive enumeration of the set $\Sigma_{0}^{\infty} j\left(t_{n}, v\left(a_{n}\right)\right)$.

Since $t_{n}$ is a regressive function of $n$ and $f(x)$ is a strictly increasing recursive function it readily follows that $t_{f(n)}$ is also a regressive function of $n$. Let $p(x)$ be any regressing function of $t_{f(n)}$. We observe here that for every number $n, p^{*}\left(t_{f(n)}\right)=n$. Let $\sigma=j(\delta p, \varepsilon)$ and for $x \in \sigma$ define $q(x)$ by,

$$
q(x)=\left\{\begin{array}{lll}
x, & \text { for } & p^{*} k(x)=0 \& l(x)=0 \\
j\left(p k(x), a_{f\left(p^{*} k(x)-1\right)}-1\right), & \text { for } & p^{*} k(x)>0 \& l(x)=0 \\
j(k(x), l(x)-1), & \text { for } & l(x)>0
\end{array}\right.
$$

Clearly $\sigma$ is r.e. and therefore $q(x)$ is a partial recursive function. Since $\varrho t_{f} \subset \delta p$ it follows that $\Sigma_{0}^{\infty} j\left(t_{n}, v\left(a_{n}\right)\right) \subset \delta q$. It is readily seen that $q(x)$ is a regressing function for the above enumeration of $\Sigma_{0}^{\infty} j\left(t_{n}, v\left(a_{n}\right)\right)$.

THEOREM 2. Let $a_{n}$ and $b_{n}$ be recursive functions. Then for every regressive isol $T$,

$$
\sum_{T}\left(a_{n}+b_{n}\right)=\sum_{T} a_{n}+\sum_{T} b_{n} .
$$

Proof. If $T$ is finite the statement is clear. Now assume that $T$ is infinite. Let $t_{n}$ be any regressive function ranging over a set in $T$. If for each $n$ we let $\sigma_{n}=\left\{x+a_{n} \mid x<b_{n}\right\}$, then 


$$
\begin{aligned}
\sum_{0}^{\infty} j\left(t_{n}, v\left(a_{n}\right)\right) & \in \sum_{T} a_{n}, \\
\sum_{0}^{\infty} j\left(t_{n}, v\left(b_{n}\right)\right) & \in \sum_{T} b_{n}, \\
\sum_{0}^{\infty} j\left(t_{n}, v\left(a_{n}+b_{n}\right)\right) & \in \sum_{T}\left(a_{n}+b_{n}\right), \\
\sum_{0}^{\infty} j\left(t_{n}, v\left(a_{n}+b_{n}\right)\right) & =\sum_{0}^{\infty} j\left(t_{n}, v\left(a_{n}\right)\right)+\sum_{0}^{\infty} j\left(t_{n}, \sigma_{n}\right) .
\end{aligned}
$$

Therefore in order to complete the proof, it suffices to prove

$$
\begin{array}{r|l}
\sum_{0}^{\infty} j\left(t_{n}, v\left(a_{n}\right)\right) \mid & \sum_{0}^{\infty} j\left(t_{n}, \sigma_{n}\right), \\
\sum_{0}^{\infty} j\left(t_{n}, v\left(a_{n}\right)\right) \cong \sum_{0}^{\infty} j\left(t_{n}, \sigma_{n}\right) .
\end{array}
$$

Let $p(x)$ be a regressing function of $t_{n}$. Set

and

$$
\delta=\left\{j(x, y) \mid x \in \delta p \quad \& \quad y<a_{p^{*}(x)}\right\}
$$

$$
\eta=\left\{j(x, y) \mid x \in \delta p \quad \& \quad y \geqq a_{p^{*}(x)}\right\} .
$$

Then $\delta$ and $\eta$ are disjoint r.e. sets with

$$
\sum_{0}^{\infty} j\left(t_{n}, v\left(a_{n}\right)\right) \subset \delta \quad \text { and } \quad \sum_{0}^{\infty} j\left(t_{n}, \sigma_{n}\right) \subset \eta .
$$

This verifies (5). Concerning (6), let

$$
q(x)=j\left(k(x), l(x)+a_{p^{*} k(x)}\right), \quad \text { for } x \in j(\delta p, \varepsilon) .
$$

Clearly $q(x)$ is a partial recursive function. Also, if $q(x)=q(y)$ for some $x$ and $y$ belonging to $\delta q$, then

$$
\begin{aligned}
q(x)=q(y) & \Rightarrow k(x)=k(y) \& l(x)+a_{p^{* k}(x)}=l(y)+a_{p^{*} k(y)} \\
& \Rightarrow k(x)=k(y) \& l(x)=l(y) \\
& \Rightarrow \quad x=y .
\end{aligned}
$$

Therefore $q(x)$ is also one-to-one. It now readily follows that

$$
\sum_{0}^{\infty} j\left(t_{n}, v\left(b_{n}\right)\right) \subset \delta q \quad \text { and } \quad q: \sum_{0}^{\infty} j\left(t_{n}, v\left(b_{n}\right)\right) \rightarrow \sum_{0}^{\infty} j\left(t_{n}, \sigma_{n}\right) .
$$

This verifies (6) and completes the proof of Theorem 2.

We obtain the following as an immediate corollary of Theorem 2. 
COROLLARY 1. Let $a_{n}$ and $b_{n}$ be two recursive functions such that for all $n, a_{n} \leqq b_{n}$. Then for all regressive isols $T$,

$$
\sum_{T}\left(b_{n}-a_{n}\right)=\sum_{T} b_{n}-\sum_{T} a_{n} .
$$

Notation. Let

$$
\underbrace{a_{0}+a_{1}+a_{2}+\ldots}_{T} \text { denote } \sum_{T} a_{n} .
$$

Proposition 1. Let $a_{n}$ be a recursive function and $u$ any number. Then for every regressive isol $T, T+1$ is also a regressive isol and

$$
\underbrace{u+a_{0}+a_{1}+a_{2}+\ldots}_{T+1}=u+\underbrace{a_{0}+a_{1}+a_{2}+\ldots}_{T} .
$$

Proof. Left to the reader.

\section{The extensions $C_{f}(X)$ and $D_{f}(X)$.}

With every recursive function $f(x)$, Myhill [6] associates two specific recursive combinatorial functions $f^{+}(x)$ and $f^{-}(x)$, called the positive and negative parts of $f(x)$, respectively. These functions have the property that for all $x, f(x)=f^{+}(x)=f-(x)$. The Nerode extension of $f(x)$ to $A$ may then be given by

$$
D_{f}(X)=C_{f+}(X)-C_{f^{-}}(X), \quad \text { for } X \in \Lambda .
$$

It can be proven that if $f^{+}(x)$ and $f-(x)$ are any two recursive combinatorial functions, such that for all $x$

then

$$
f(x)=f^{+}(x)-f^{-}(x),
$$

$$
D_{f}(X)=C_{f+}(X)-C_{f-}(X), \quad \text { for } X \in \Lambda .
$$

In this section we wish to give a representation of $D_{f}(T)$ (for $f$ a recursive function and $T$ a regressive isol) as the difference of two infinite series, each summed with respect to the regressive isol $T+1$. In view of (7) it will be sufficient to describe a method whereby $C_{f}(T)$ (for $f$ a recursive combinatorial function and $T$ a regressive isol) can be expressed as an infinite series summed with respect to $T+1$.

Definition. Let $f(x)$ be a function from $\varepsilon$ into $\varepsilon$. The functions

$$
\begin{aligned}
d(n) & =f(n+1)-f(n), \\
e(0) & =f(0), \\
e(n+1) & =d(n),
\end{aligned}
$$


are the $d$-difference and e-difference functions of $f(x)$, respectively. We shall also write $d_{n}$ for $d(n)$ and $e_{n}$ for $e(n)$.

It is an easy consequence of the above definition that the $d$-difference and $e$-difference functions of recursive functions are also recursive. In addition, if $f(x)$ is recursive and combinatorial, then so is $d(x)$ and we have for all $x$,

$$
f(x)=f(0)+\sum_{n<x} d_{n} .
$$

TheOREM 3. Let $f(x)$ be a recursive combinatorial function. Let $d(x)$ be the d-difference function of $f(x)$. Then for all regressive isols $T$,

$$
C_{f}(T)=f(0)+\sum_{T} d_{n} .
$$

Proof. Let $s(n)$ be the partial sum function of $d(n)$. Since $d(n)$ is recursive and combinatorial, so is $s(n)$. Also, we have for all $x$

$$
\begin{aligned}
s(x) & =\sum_{n<x} d_{n}, \\
f(x) & =f(0)+\sum_{n<x} d_{n}, \\
f(x) & =f(0)+s(x) .
\end{aligned}
$$

Identity (10) concerns only recursive combinatorial functions and therefore yields by a well-known theorem of Myhill

$$
C_{f}(X)=f(0)+C_{s}(X), \quad \text { for } X \in \Lambda .
$$

By applying Theorem A to (8) we obtain

$$
C_{s}(T)=\sum_{T} d_{n}, \quad \text { for } T \in \Lambda_{R} .
$$

Combining (11) and (12) we obtain the desired result

$$
C_{f}(T)=f(0)+\Sigma_{T} d_{n}, \quad \text { for } T \in \Lambda_{R} .
$$

CoRollary 2. Let $f(x)$ be a recursive combinatorial function and $e(x)$ its e-difference function. Then for all regressive isols $T$,

$$
C_{f}(T)=\sum_{T+1} e_{n} .
$$

Proof. Use Theorem 3, Proposition 1 and the definition of $e_{n}$.

Corollary 3. Let $f(x)$ be a recursive function. Let $f^{+}(x)$ and $f^{-}(x)$ be any two recursive combinatorial functions such that, for all $x, f(x)=$ $f^{+}(x)-f^{-}(x)$. Let $e^{+}(x)$ and $e^{-(x)}$ be the e-difference functions of $f^{+}(x)$ and $f^{-}(x)$, respectively. Then for all regressive isols $T$,

$$
D_{f}(T)=\sum_{T+1} e_{n}^{+}-\sum_{T+1} e_{n^{-}} .
$$


Proof. Use (7) and Corollary 2.

LEMMA 1. Let $f(x)$ be an increasing recursive function. Let $f^{+}(x)$ and $f^{-}(x)$ be any two recursive combinatorial functions such that, for all $x$, $f(x)=f^{+}(x)-f^{-}(x)$. Let $e(x), e^{+}(x)$ and $e^{-}(x)$ denote the e-difference functions of $f(x), f^{+}(x)$ and $f^{-}(x)$, respectively. Then for all $x$,

$$
\begin{gathered}
e^{+}(x) \geqq e^{-}(x), \\
e(x)=e^{+}(x)-e^{-}(x) .
\end{gathered}
$$

Proof. Left to the reader.

Proposition 2. Let $f(x)$ be an increasing recursive function and $e(x)$ its e-difference function. Then for all regressive isols $T$,

$$
D_{f}(T)=\sum_{T+1} e_{n} .
$$

Proof. Since every recursive function can be expressed as the difference of two recursive combinatorial functions, the desired conclusion will follow from Lemma 1, Corollary 3 and Theorem 2.

CoRollary 4. Let $f(x)$ be an increasing recursive function. Then for all regressive isols $T$,

$$
D_{f}(T) \in \Lambda_{R} \text {. }
$$

Proof. Use Proposition 2 and Theorem 1.

\section{The principal theorem.}

Let $f(x)$ and $g(x)$ be any two recursive combinatorial functions. Then it is well-known that both of the functions $[f+g](x)$ and $f g(x)$ are also recursive combinatorial and moreover for all isols $X$,

$$
\begin{aligned}
C_{f+g}(X) & =C_{f}(X)+C_{g}(X), \\
C_{f g}(X) & =C_{f}\left(C_{g}(X)\right) .
\end{aligned}
$$

We shall use (13) and (14) in proving the following two lemmas.

LEMMA 2. Let $f(x)$ be a recursive function and $h(x)$ a recursive combinatorial function. Then

$$
D_{f h}(X)=D_{f}\left(C_{h}(X)\right), \quad \text { for } X \in \Lambda .
$$

Proof. Let $f^{+}(x)$ and $f^{-}(x)$ be any two recursive combinatorial functions such that for all $x$,

$$
f(x)=f^{+}(x)-f^{-}(x) .
$$


Since $h(x)$ is recursive and combinatorial, so are $f^{+} h(x)$ and $f^{-} h(x)$, and for all $x$,

$$
f h(x)=f^{+} h(x)-f-h(x) .
$$

From (7) it now follows that for all isols $X$,

$$
\begin{gathered}
D_{f}(X)=C_{f+}(X)-C_{f-}(X), \\
D_{f h}(X)=C_{f+h}(X)-C_{f-h}(X) .
\end{gathered}
$$

In view of (14), (17) yields

$$
D_{f h}(X)=C_{f+}\left(C_{h}(X)\right)-C_{f-}\left(C_{h}(X)\right), \quad \text { for } X \in \Lambda .
$$

We can now obtain (15) from (16) and (18). This completes the proof of Lemma 2.

Lemma 3. Let $f(x)$ be a recursive function. Let $f^{\wedge}(x)$ and $f^{\curlyvee}(x)$ be any two recursive functions such that

Then

$$
f(x)=f^{\wedge}(x)-f^{`}(x), \quad \text { for } \quad x \in \varepsilon .
$$

$$
D_{f}(X)=D_{f^{\wedge}}(X)-D_{f^{-}}(X), \quad \text { for } X \in \Lambda \text {. }
$$

Proof. Let $g^{+}(x)$ and $g^{-}(x)$, and $h^{+}(x)$ and $h^{-}(x)$ be any two pairs of recursive combinatorial functions such that, for all $x$

$$
f^{\wedge}(x)=g^{+}(x)-g^{-}(x) \text { and } f^{\curlyvee}(x)=h^{+}(x)-h^{-}(x) .
$$

It readily follows that $\left[g^{+}+h^{-}\right](x)$ and $\left[g^{-}+h^{+}\right](x)$ are also recursive combinatorial functions and such that, for all $x$

$$
f(x)=\left[g^{+}+h^{-}\right](x)-\left[g^{-}+h^{+}\right](x) .
$$

In view of (7), (20) and (21) imply that for all isols $X$,

$$
\begin{aligned}
D_{f}(X) & =C_{g^{+}}(X)-C_{g^{-}}(X), \\
D_{f}(X) & =C_{h^{+}}(X)-C_{h^{-}}(X), \\
D_{f}(x) & =C_{g^{++h^{-}}}(X)-C_{g^{-+h^{+}}}(x) .
\end{aligned}
$$

Application of (13) to (24) yields

$$
D_{f}(X)=\left[C_{g^{+}}(X)+C_{h^{-}}(X)\right]-\left[C_{g^{-}}(X)+C_{h^{+}}(X)\right], \quad \text { for } X \in \Lambda .
$$

Algebraic rearrangement of (25) together with (22) and (23) give (19). This completes the proof of Lemma 3.

THEOREM 4. Let $f(x)$ be a recursive function. Then the following conditions are equivalent. 

$f(x)$ is an eventually increasing recursive function. $D_{f}(X)$ maps $\Lambda_{R}$ into $\Lambda_{R}$.

Proof. We first prove that (26) implies (27). Let $f(x)$ be an eventually increasing recursive function. Obviously for every finite regressive isol $t$, $D_{f}(t)$ is finite and hence also regressive.

Suppose now $T$ is an infinite regressive isol. Let $n$ be a number such that $g(x)=f(x+n)$ is an increasing recursive function. Both $f(x)$ and $g(x)$ are recursive functions. Also, it is well-known that $x+n$ is a recursive combinatorial function of $x$ with canonical extension $X+n$. We can now conclude from Lemma 2, that

$$
D_{g}(X)=D_{f}(X+n), \quad \text { for } X \in \Lambda .
$$

Since $T$ is an infinite regressive isol, so is $T-n$. Substitution in (28) gives

$$
D_{g}(T-n)=D_{f}(T) .
$$

In view of Corollary $4, D_{f}(T)$ is a regressive isol. This gives (27) and completes half of the proof.

We shall prove that (27) implies (26) by proving the following statement.

$$
\left\{\begin{array}{l}
\text { Let } f(x) \text { be a recursive function which is not eventu- } \\
\text { ally increasing. Then there exists a regressive isol } T \\
\text { such that } D_{f}(T) \text { is not an isol, that is } D_{f}(T) \in \Lambda^{*}-\Lambda .
\end{array}\right.
$$

Let $f(x)$ be a recursive function which is not eventually increasing. Define the functions

$$
\begin{aligned}
\Delta(0) & =f(0), \\
\Delta(n) & =f(n)-f(n-1) \quad \text { for } n>0, \\
a_{n} & =\max (0, \Delta(n)), \\
b_{n} & =\max (0,-\Delta(n)) .
\end{aligned}
$$

$\Delta(n)$ will not map $\varepsilon$ into $\varepsilon$. However, it readily follows from the recursiveness of $f(x)$ that $a_{n}$ and $b_{n}$ are recursive functions of $n$. Also, it follows that

$$
\begin{gathered}
(\forall n)\left[\min \left(a_{n}, b_{n}\right)=0\right], \\
(\forall x)\left[f(x)=\sum_{n \leqq x} a_{n}-\sum_{n \leqq x} b_{n}\right] .
\end{gathered}
$$

Set

$$
f^{\wedge}(x)=\sum_{n \leqq x} a_{n} \quad \text { and } \quad f^{\vee}(x)=\sum_{n \leqq x} b_{n} .
$$


Then $f^{\wedge}(x)$ and $f^{\curlyvee}(x)$ are increasing recursive functions such that, for all $x$

$$
f(x)=f^{\wedge}(x)-f^{\curvearrowright}(x) .
$$

By Lemma 3, (33) yields

$$
D_{f}(X)=D_{f^{\wedge}}(X)-D_{f^{\wedge}}(X), \quad \text { for } X \in \Lambda .
$$

$f^{\wedge}(x)$ and $f^{\curvearrowright}(x)$ are increasing recursive functions; in view of their definitions they will have $a_{n}$ and $b_{n}$ as their respective $e$-difference functions. Therefore (34) and Proposition 2 imply

$$
D_{f}(T)=\sum_{T+1} a_{n}-\sum_{T+1} b_{n}, \quad \text { for } T \in \Lambda_{R} .
$$

It now follows that in order to prove statement (30), it suffices to prove the existence of a regressive isol $T$, such that

$$
\operatorname{not}\left[\sum_{T+1} a_{n} \geqq \sum_{T+1} b_{n}\right] \text {. }
$$

This will be our approach here and we shall use a technique introduced in the proof of [3, Theorem 95].

We first observe that the recursive functions $a_{n}$ and $b_{n}$ are each positive infinitely often. This follows from (32) and the fact that the function $f(x)$ is recursive and not eventually increasing. Therefore the sets $\left\{n \mid a_{n}>0\right\}$ and $\left\{n \mid b_{n}>0\right\}$ are each infinite and recursive, and by (31) are disjoint. Let $g(n)$ and $h(n)$ denote the strictly increasing recursive functions ranging over $\left\{n \mid a_{n}>0\right\}$ and $\left\{n \mid b_{n}>0\right\}$, respectively.

Let $p_{i}(x)$ be a function of the two variables $i$ and $x$ not necessarily everywhere defined such that every one-to-one partial recursive function, and no other function, appears in the sequence $\left\{p_{i}\right\}$. We shall now define a regressive function $t_{n}$, such that if $T+1=\operatorname{Req}(\varrho t)$, then $T$ will be an infinite regressive isol satisfying (36).

Set $t_{0}=1$. Assuming that $t_{0}, \ldots, t_{k-1}$ with $k \geqq 1$ have already been defined, we define $t_{k}$ by setting

$$
t_{k}=j\left(t_{k-1}, u_{k}\right),
$$

where $u_{k}$ is determined as follows:

Case 1: $k \notin \varrho g$ and $k \notin \varrho h$. Set $u_{k}=0$.

Case 2: $k \in \varrho g$, say $k=g(i)$. Let $h(0), \ldots, h(s)$ be all those $h(r)$ 's with $h(r)<g(i)$. Now put $u_{k}$ equal to the smallest number $w$ such that for each $v$ with $0 \leqq v<a_{g(i)}$,

$$
j\left(j\left(t_{k-1}, w\right), v\right) \notin\left(p_{0} j\left(t_{h(0)}, 0\right), \ldots, p_{s} j\left(t_{h(s)}, 0\right)\right) .
$$

Case 3: $k \in \varrho h$, say $k=h(i)$. Let $g(0), \ldots, g(s)$ be all those $g(r)$ 's with $g(r)<h(i)$. Now put $u_{k}$ equal to the smallest number $w$ such that either 


$$
p_{i} j\left(j\left(t_{k-1}, w\right), 0\right) \text { is undefined },
$$

or $p_{i} j\left(j\left(t_{k-1}, w\right), 0\right)$ is defined and

$$
p_{i} j\left(j\left(t_{k-1}, w\right), 0\right) \notin\left\{j\left(t_{g(n)}, v\right) \mid n \leqq s \& v<a_{g(n)}\right\} .
$$

The existence of $u_{k}$ in Case 2 follows because $j$ maps $\varepsilon^{2}$ one-to-one onto $\varepsilon$. We obtain the existence of $u_{k}$ in Case 3 because each of the functions $p_{i}$ is one-to-one.

Let

$$
\tau=\varrho t, \quad \tau^{\prime}=\tau-\left(t_{0}\right) \quad \text { and } \quad T=\operatorname{Reg}\left(\tau^{\prime}\right) .
$$

To complete the proof we will verify:

(i) $t_{n}$ is a regressive function,

(ii) $T$ and $T+1$ are regressive R.E.T.s,

(iii) $T$ satisfies (36),

(iv) $T$ is an isol.

Re (i). It follows from

$$
j(x, y)=\frac{1}{2}(x+y)(x+y+1)+x
$$

that $x<j(x, y)$ for $x>0$. By the definition of $t$ we have

$$
t_{0}>0 \quad \text { and } \quad(\forall n)(\exists u)\left[t_{n+1}=j\left(t_{n}, u\right)\right]
$$

Hence $t_{0}<t_{1}<t_{2}<\ldots$, and therefore $t$ is a one-to-one function. Let

$$
g(x)=\left\{\begin{array}{lll}
t_{0}, & \text { for } & x=t_{0} \\
k(x), & \text { for } & x \neq t_{0}
\end{array}\right.
$$

It readily follows that $g(x)$ is a regressing function of $t_{n}$. Hence $t_{n}$ is a regressive function.

$\operatorname{Re}(i i)$. Set $t^{\prime}{ }_{n}=t_{n+1}$. Then $t_{n}^{\prime}$ is obviously also a regressive function. Since $\tau=\varrho t$ and $\tau^{\prime}=\varrho t^{\prime}, \tau$ and $\tau^{\prime}$ are regressive sets. Also, $\tau^{\prime} \in T$ and $\tau \in T+1$, and therefore $T$ and $T+1$ are regressive R.E.T.s.

Re (iii). Since $t_{n}$ is a regressive function ranging over a set in $T+1$, we have

$$
\begin{aligned}
& \sum_{0}^{\infty} j\left(t_{n}, v\left(a_{n}\right)\right) \in \sum_{T+1} a_{n}, \\
& \sum_{0}^{\infty} j\left(t_{n}, v\left(b_{n}\right)\right) \in \sum_{T+1} b_{n} .
\end{aligned}
$$

In addition, since the functions $g(n)$ and $h(n)$ range over all the positive $a_{n}$ and $b_{n}$, respectively, it follows that 


$$
\begin{aligned}
& \sum_{0}^{\infty} j\left(t_{n}, v\left(a_{n}\right)\right)=\sum_{0}^{\infty} j\left(t_{g(n)}, v\left(a_{g(n)}\right)\right), \\
& \sum_{0}^{\infty} j\left(t_{n}, v\left(b_{n}\right)\right)=\sum_{0}^{\infty} j\left(t_{h(n)}, v\left(b_{h(n)}\right)\right) .
\end{aligned}
$$

We wish to prove that $T$ satisfies (36). Let us suppose otherwise, namely

$$
\sum_{T+1} b_{n} \leqq \sum_{T+1} a_{n} .
$$

Then in view of (37), (38), (39) and (40) it follows that there would exist a one-to-one partial recursive function $p(x)$, such that

$$
\begin{gathered}
\sum_{0}^{\infty} j\left(t_{h(n)}, v\left(b_{h(n)}\right)\right) \subset \delta p, \\
p\left(\sum_{0}^{\infty} j\left(t_{h(x)}, v\left(b_{h(n)}\right)\right)\right) \subset \sum_{0}^{\infty} j\left(t_{g^{\prime}(n)}, v\left(a_{g(n)}\right)\right) .
\end{gathered}
$$

We shall prove that this is not possible. Suppose the index of $p(x)$ in our enumeration of all one-to-one partial recursive functions is $i$, that is, $p(x)=p_{i}(x)$. Then from (42) and (43) we would have in particular for some number $r$,

$$
\begin{gathered}
j\left(t_{h(i)}, 0\right) \in \delta p_{i}, \\
p_{i} j\left(t_{h(i)}, 0\right) \in\left(j\left(t_{g(r)}, 0\right), \ldots, j\left(t_{g(r)}, a_{g(r)}-1\right)\right) .
\end{gathered}
$$

The functions $g(n)$ and $h(n)$ have disjoint ranges and therefore either $h(i)<g(r)$ or $g(r)<h(i)$.

Suppose that $h(i)<g(r)$. By Case 2 of the construction, $t_{g(n)}$ is so defined that for each $v$ with $0 \leqq v<a_{g(r)}$,

$$
j\left(t_{g(r)}, v\right) \notin\left\{p_{n} j\left(t_{h(n)}, 0\right) \mid h(n)<g(r)\right\} .
$$

Since $h(i)<g(r)$, this implies

$$
p_{i} j\left(t_{h(i)}, 0\right) \notin\left(j\left(t_{g(r)}, 0\right), \ldots, j\left(t_{g(r)}, a_{g(r)}-1\right)\right),
$$

which would be false in view of (45). Therefore $h(i)<g(r)$ would not be possible.

Suppose now that $g(r)<h(i)$. By Case 3 of the construction, $t_{h(i)}$ is so defined that either $p_{i} j\left(t_{h(i)}, 0\right)$ is undefined or $p_{i} j\left(t_{h(i)}, 0\right)$ is defined and

$$
p_{i} j\left(t_{h(i)}, 0\right) \notin\left\{j\left(t_{g(n)}, v\right) \mid g(n)<h(i) \& v<a_{g(n)}\right\} .
$$

In view of $(44)$ we may assume that $p_{i} j\left(t_{h(i)}, 0\right)$ is defined. Since $g(r)<$ $h(i),(46)$ yields 


$$
p_{i} j\left(t_{h(i)}, 0\right) \notin\left(j\left(t_{g(r)}, 0\right), \ldots, j\left(t_{g(r)}, a_{g(r)}-1\right)\right),
$$

which would be false in view of $(45)$. Therefore $g(r)<h(i)$ would also not be possible.

We can now conclude that there exists no one-to-one partial recursive function satisfying conditions (42) and (43). Therefore the inequality of (41) is false and $T$ will be a regressive R.E.T. satisfying (36). This completes the proof of (iii).

$R e(i v)$. An infinite set is retraceable, if it is the range of a strictly increasing regressive function. As a consequence, the function $t_{n}$ mentioned in the proof of $(i)$ is a strictly increasing regressive function, and hence $\tau$ is a retraceable set. It is proved in [4] that every retraceable set is either recursive or immune.

We now prove that $\tau$ is not a recursive set. Suppose otherwise, then the strictly increasing function ranging over $\tau$, namely $t_{n}$, would be recursive. Since $g(n)$ and $h(n)$ are also recursive functions, it would then be an easy consequence that

$$
\sum_{0}^{\infty} j\left(t_{g(n)}, v\left(a_{g(n)}\right)\right) \quad \text { and } \quad \sum_{0}^{\infty} j\left(t_{h(n)}, v\left(b_{h(n)}\right)\right)
$$

are infinite r.e. sets. However, then these sets would be recursively equivalent, contrary to our above remarks.

Since $\tau$ is retraceable and not recursive, $\tau$ is immune. Since $\tau \in T+1$, it follows that $T+1$ and hence also $T$ is an isol. This verifies (iv) and completes the proof of Theorem 4 .

\section{REFERENCES}

1. J. C. E. Dekker, Infinite series of isols, Amer. Math. Soc. Proc. of the Symposia in Pure Mathematics 5 (1962), 77-96.

2. J. C. E. Dekker, The minimum of two regressive isols, Math. Z. 83 (1964), 345-366.

3. J. C. E. Dekker and J. Myhill, Recursive equivalence types, Univ. California Publ. Math. (N. S.) 3 (1960), 67-214.

4. J. C. E. Dekker and J. Myhill, Retraceable sets, Canad. J. Math. 10 (1958), 357-373.

5. J. Myhill, Recursive equivalence types and combinatorial functions, Bull. Amer. Math. Soc. 64 (1958), 373-376.

6. J. Myhill, Recursive equivalence types and combinatorial functions, Proc. of the International Congress in Logic and Methodology of Science, Stanford (1960), 46-55.

7. A. Nerode, Extensions to isols, Ann. of Math. 73 (1961), 362-403.

8. A. Nerode, Extensions to isolic integers, Ann. of Math. 75 (1962), 419-448.

RUTGERS, THE STATE UNIVERSITY, NEW BRUNSWICK, NEW JERSEY, U.S.A.

UNIVERSITY OF PITTSBURGH, PITTSBURGH, PENNSYlVANIA, U.S.A. 